\title{
Aproximación al manejo del dolor en la enfermedad ósea metastásica
}

\author{
Approach to the management of pain in metastatic bone disease
}

\author{
- Ana Antolínez P.', Pedro Pérez S. ', Diego González R. ${ }^{1}$ \\ 'Especialista en entrenamiento de Medicina del Dolor y Cuidados Paliativos, Universidad Militar Nueva Granada, Instituto Nacional de Cancerología (Bogotá, Colombia).
}

\section{Resumen}

Introducción: La enfermedad ósea metastásica es una condición frecuente y devastadora para el enfermo oncológico en términos de disminución de la funcionalidad, autonomía, dolor y reducción de la calidad de vida. El dolor óseo secundario a enfermedad metastásica tiene una etiología multifactorial compleja y se considera de difícil manejo. A pesar de la existencia de múltiples herramientas terapéuticas para esta condición, persiste un grupo importante de pacientes que no reciben un manejo optimizado de dolor.

Objetivos: El objetivo de este artículo es revisar las diferentes estrategias para el manejo del dolor óseo secundario a enfermedad metastásica.

Materiales y métodos: Se realizó una búsqueda de la literatura en PubMed, ScienceDirect y MD Consult, con palabras MeSH y texto libre a partir de 1989. Se obtuvieron 211 artículos y se seleccionaron 80 por su relevancia.

Conclusiones: El dolor óseo en la enfermedad ósea metastásica se considera de etiología multifactorial y de difícil manejo; existen diferentes estrategias terapéuticas para su tratamiento, recomendándose la aplicación de la analgesia multimodal. El especialista en dolor tiene un papel fundamental en la orientación del manejo no solamente farmacológico, sino en la estructuración de un tratamiento multidisciplinario coordinado encaminado al logro de los objetivos.

Palabras clave: Metástasis ósea, bifosfonatos, manejo de dolor, analgésicos, analgésicos opioides, anticonvulsivantes, radioterapia, radioisótopos.

\begin{abstract}
Introduction: Metastatic bone disease is a common and devastating condition for cancer patients in terms of reduced functionality, autonomy, pain and decreased quality of life. Bone pain induced by cancer metastases has a complex multifactorial etiology and is considered difficult pain management. Despite the existence of multiple therapeutic tools for this condition, a large group of patients did not receive appropriate pain management.

Objectives: The aim of this article is to review the different strategies for management of bone pain secondary to metastatic disease. Materials and methods: We performed a literature search in PubMed, ScienceDirect and MD Consult, with MeSH words and free text from 1989 where 211 articles were obtained. 80 of these articles were selected due to their relevance.

Conclusions: Bone pain in metastatic bone disease etiology is considered multifactorial, and difficult to manage; there are different therapeutic strategies for management; multimodal analgesia strategy is widely recommended. Pain specialist plays a key role not only in guiding the pharmacological management but in structuring a coordinated multidisciplinary approach aimed at achieving the objectives.
\end{abstract}

Keywords (MeSH): Bone metastases, bisphosphonates, pain management, analgesics, analgesics opioids, anticonvulsants, radiotherapy, radioisotopes.

\section{Generalidades}

La enfermedad metastásica ósea representa la mayor parte de lesiones óseas en los pacientes mayores de 40 años de edad. De hecho, el diagnóstico diferencial de una lesión ósea agresiva en un adulto es metástasis, mieloma, linfoma y sarcoma, en orden decreciente de frecuencia. Incluso, las lesiones óseas solitarias en este grupo de edad son debidas con mayor frecuencia a un carcinoma metastásico'.

A nivel mundial se estima que aproximadamente un $30-50 \%$ de pacientes con cáncer experimentan dolor $^{2,3}$, esta prevalencia puede llegar hasta el $60-90 \%$ en algunos tipos específicos de cáncer, como son los tumores de cabeza y cuello, genitourinarios, páncreas

Recibido: 16/06/2015. Aceptado: 14/01/2016

Derechos de reproducción: @ 2016 Antolínez et al. Este es un artículo de acceso libre distribuido bajo los términos de la licencia Creative Commons BY-NC-ND, la cual otorga permiso público para compartir y usar los artículos bajo la condición de atribución al autor, no modificación de su contenido y no uso con fines comerciales

Este artículo debe citarse como Antolínez A, Pérez P. González D. Aproximación al manejo del dolor en la enfermedad ósea metastásica. Revista Colombiana de Hematología y Oncología. 2016;3(1):24-32. 
y próstata ${ }^{4}$; en estadios avanzados o terminales de la enfermedad, se reportan prevalencias hasta del $70 \%$ y en supervivientes de cáncer se considera una prevalencia del $33 \% \%^{5}$. Existen múltiples causas de dolor en este grupo poblacional, pero es la enfermedad ósea metastásica su causa más común, representando del 60 al 80\% $\%^{6,7}$, que además ocasiona complicaciones, como hipercalcemia, y eventos esqueléticos, como fractura patológica y compresión medular; el 70\% de los pacientes clasifican su dolor óseo como moderado a severo con importante impacto en funcionalidad y calidad de vida ${ }^{8}$

El dolor oncológico no controlado es uno de los principales síntomas que afectan de forma negativa la salud física y psicológica de los pacientes con diagnóstico de cáncer ${ }^{9}$, situación que es especialmente dramática debido a que existe evidencia sobre los múltiples efectos benéficos del control de dolor ${ }^{10}$; sin embargo, aún hay una importante brecha entre la evidencia clínica y la práctica. Se consideran múltiples barreras a las que se atribuye este desequilibrio, una de ellas es el inadecuado entrenamiento de los trabajadores de salud en cuanto a la valoración y medición del dolor con instrumentos validados $^{11}$, así como su manejo farmacológico ${ }^{12,13}$, lo que explica que hasta un tercio de esta población reciba analgésicos comunes o débiles 0 , incluso, no reciban manejo farmacológico para el control del síntoma ${ }^{14-16}$.

La severidad del dolor óseo no necesariamente se relaciona con la magnitud del compromiso; así mismo, el dolor puede o no estar presente desde el inicio de la enfermedad ${ }^{17}$, y es considerado uno de los síntomas más incapacitantes para estos pacientes ${ }^{18}$. La caracterización del dolor es variable, pero, en general, se describe en términos de temporalidad como constante y en términos de calidad como un dolor somático (profundo); hay una asociación frecuente con dolor episódico, que puede estar relacionado con un evento desencadenante claro, como la movilización del segmento afectado, o puede no asociarse a un evento, en cuyo caso puede ser el resultado de la remodelación ósea frecuente y de microfracturas ${ }^{19}$.

\section{Fisiopatología}

En cuanto a su fisiopatología, inicialmente se consideró que el dolor de la enfermedad ósea metastásica tenía un origen inflamatorio o neuropático; sin embargo, estudios en modelos murinos muestran patrones claramente diferenciados, tanto conductuales como neuroquímicos, en la médula espinal y neuronas sensoriales, lo que sugiere la coexistencia de diferentes tipos de dolor que persisten y cambian a medida que evoluciona la enfermedad ${ }^{20}$. Anatómicamente en el hueso los nervios acompañan a los vasos sanguíneos nutricios; el periostio tiene una rica inervación sensitiva, que explica el dolor generado por la deformación ósea y fractura, a diferencia del tejido óseo propiamente dicho, donde predominan nervios simpáticos encargados del control vasomotor de tejido óseo y médula ósea ${ }^{21}$.

El origen fisiopatológico del dolor óseo en la enfermedad metastásica es multifactorial, en el que participan sustancias producidas por células tumorales, células inflamatorias y células propias del tejido óseo. Por un lado, están los estímulos mecánicos sobre nociceptores (neurona sensitiva aferente de primer orden), ocasionados directamente por el efecto de masa sobre el periostio y sus fibras nerviosas, así como sobre las estructuras adyacentes; por otro lado, nos encontramos con toda una cascada de elementos pro inflamatorios, algogénicos y factores de crecimiento que se encuentran en estrecha relación con la fisiopatología de la enfermedad metastásica propiamente dicha. El receptor vaniloide (RV), ubicado en el nociceptor, es activado por la caída en el pH debido al aumento en la concentración de hidrogeniones, lo que puede ocurrir tanto por el daño tisular y la apoptosis asociado al cáncer como por la activación de células inflamatorias.

Los osteoclastos durante su proceso de activación disminuyen el pH pericelular, lo cual puede contribuir al aumento del dolor; otras sustancias que incrementan la actividad de estos nociceptores son el calor, los lípidos y la capsaicina ${ }^{22}$. La activación de receptores de endotelina implicada en la génesis del dolor acrecienta la

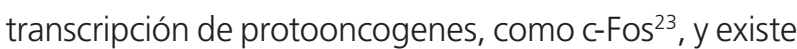
evidencia indirecta de que su modulación podría impactar de forma positiva en el tratamiento del cáncer ${ }^{24}$; los inhibidores de endotelina han sido evaluados en ensayos clínicos fase III en cáncer de próstata resistente a la terapia de deprivación hormonal, sin embargo, no se ha demostrado aumento en la supervivencia global al adicionarlo a tratamiento con docetaxe ${ }^{25}$. Otros receptores con actividad de nociceptor son: Drasic (the dorsal-root acid-sensing ion channel), los cuales se activan por cambios en la tensión del tejido y el receptor de prostaglandina activado por derivados del ácido 
araquidónico ampliamente utilizado para el control del dolor agudo (tabla 1).

Todas estas sustancias implicadas en la alteración del metabolismo óseo son también responsables de la neoproliferación de estructuras nerviosas y sobreexpresión de receptores en la membrana postsináptica, que son causantes de la sensibilización central, lo cual conlleva estados crónicos de dolor ${ }^{26,27}$. Esta reorganización neuroquímica en los segmentos espinales incluye la hipertrofia de células gliales (astrocitos), disminución de la expresión de transportadores (recaptación) de glutamato, dando como resultado un exceso de este neurotransmisor a nivel de la sinapsis. A todo este conjunto de modificaciones se ha denominado toxicidad excitatoria.

Tabla 1. Localización y producción de agonistas de nociceptores

\begin{tabular}{|l|l|l|}
\multicolumn{1}{|c|}{$\begin{array}{c}\text { Localización/ } \\
\text { nociceptor }\end{array}$} & \multicolumn{1}{|c|}{$\begin{array}{c}\text { Sustancia } \\
\text { algógena }\end{array}$} & \multicolumn{1}{c|}{$\begin{array}{c}\text { Célula } \\
\text { productora }\end{array}$} \\
\hline $\begin{array}{l}\text { Periostio/mixto } \\
\text { (purinérgicos, promiscuos) }\end{array}$ & Sustancia P, CGRP, ATP & $\begin{array}{l}\text { Células tumorales, } \\
\text { tejido óseo }\end{array}$ \\
\hline Periostio/mixto & $\begin{array}{l}\text { Prostaglandinas, endote- } \\
\text { linas, bradicinina, factor } \\
\text { estimulador de colonias, } \\
\text { TNF-a, PDGF, IL-1, NGF, IL-6 }\end{array}$ & $\begin{array}{l}\text { Osteoclastos, osteoblastos, } \\
\text { células tumorales, células } \\
\text { inmunes }\end{array}$ \\
\hline $\begin{array}{l}\text { Región tumoral/TRPV1 } \\
\text { y ASIC3 }\end{array}$ & Acidosis & $\begin{array}{l}\text { Osteoclastos y células } \\
\text { tumorales }\end{array}$ \\
\hline
\end{tabular}

\section{Manejo del dolor óseo metastásico}

El manejo del dolor óseo metastásico debe tener como base el uso de terapias que produzcan alivio significativo del dolor, disminución de la interferencia del dolor en actividades diarias, reducción de otros síntomas relacionados con el dolor, tales como alteraciones del ánimo, fatiga y disturbios del sueño, procurando el menor riesgo de efectos adversos y secundarios, con efecto analgésico de inicio rápido y duradero, a un costo razonable.

El manejo farmacológico del dolor en enfermedad ósea metastásica se corresponde con su fisiopatología multifactorial; por lo tanto, incluye diversos fármacos, cada uno de ellos con diferentes objetivos terapéuticos, como disminución del tamaño tumoral, estabilización del metabolismo óseo, estabilizadores de procesos inflamatorios y neuromoduladores ${ }^{28}$. Cada uno de estos grupos farmacológicos representa una variedad de efectos adversos y efectos secundarios que limitan su uso, de allí que se prefiriera una estrategia de analgesia multimodal, la cual implica la asociación de medicamentos con diferentes mecanismos de acción, a la mínima dosis efectiva, con el fin de evitar los efectos indeseables que se producen más frecuentemente con las dosis elevadas que requerirían cada uno de ellos por separado para alcanzar el adecuado control del síntoma. Los grupos farmacológicos de utilidad en esta patología son los antiinflamatorios no esteroides, opioides, bifosfonatos, corticosteroides, agentes quimioterápicos, además de otras estrategias terapéuticas, como la radioterapia y el manejo quirúrgico.

\section{Radioterapia}

El tratamiento radioterapéutico se indica cuando existe enfermedad extensa; el efecto secundario más relevante es la supresión medular, que limita la dosis de radiación; en la radioterapia hemicorporal superior, el efecto adverso más temido es la neumonitis posradiación, en hemicuerpo inferior; los síntomas gastrointestinales como diarrea y vómito pueden llegar a ser severos. El mecanismo por el cual se obtiene el alivio de dolor no es claro, sin embargo, la disminución del tamaño tumoral a expensas de la destrucción de células tumorales permite la remodelación osteoblástica del tejido lesionado; así mismo, la reducción de la actividad osteoclástica y la lesión sobre fibras nerviosas se consideran algunos de los mecanismos plausibles ${ }^{29,30}$. Dentro de este manejo se incluye la radioterapia externa, la radioterapia hemicorporal y la aplicación de radiofármacos $^{31}$. Aproximadamente, el $40 \%$ de los pacientes remitidos a servicios de radioterapia son pacientes con enfermedad ósea metastásica derivados para el manejo paliativo de su enfermedad.

En cuanto al tratamiento con radioterapia externa fraccionada, se encontró que en el $25 \%$ de los pacientes se produce alivio completo del síntoma, y alivio del 50\% del nivel de dolor en el $41 \%$ de los pacientes. La mejoría del dolor puede verse desde la segunda semana, con un pico máximo de efecto entre la cuarta y sexta semanas del tratamiento; con un número necesario por tratar de 4,2 (CI95\%: 3,7-4,7) y un tiempo de mantenimiento del efecto hasta de 12 semanas; no se encontraron diferencias estadísticamente significativas en cuanto a la efectividad y efectos secundarios entre esquemas con diferentes dosis de fraccionamiento ${ }^{32}$. Las dosis estandarizadas de tratamiento se basan en manejo diario que va de 10 a 14 sesiones con dosis total de 30 a 35 Gy. En cuanto a radioterapia de dosis única, se considera que existe diferencia significativa con respecto 
a la tasa de respuesta en control de dolor, a favor de $8 \mathrm{~Gy}$ versus $4 \mathrm{~Gy}{ }^{33}$; en relación con la costo-efectividad, los esquemas de dosis única parecen ser más favorables que los esquemas de dosis fraccionadas ${ }^{34}$.

\section{Radiofrecuencia}

La ablación por radiofrecuencia es un procedimiento mínimamente invasivo, realizado bajo sedación consciente, guiado por imágenes, para el tratamiento de tumores sólidos, tanto en lesiones osteolíticas como osteoblásticas, en el que se aplica un estímulo térmico originado en un sistema eléctrico cerrado, directamente sobre la lesión, produciendo cambios citotóxicos celulares inmediatos que ocasionan necrosis tumoral; en general, se reserva para pacientes con contraindicación o refractarios al tratamiento convencional con radioterapia, o aquellos que tienen enfermedad localizada, como metástasis única. Se ha evidenciado mejoría significativa en el control de dolor hasta en el $95 \%$ de los pacientes tratados, con mantenimiento del resultado hasta 24 semanas posterior al procedimiento ${ }^{35,36}$. La complicación que más se ha reportado es la necrosis cutánea localizada, aunque raramente ocurre.

\section{Medicamentos analgésicos y adyuvantes}

Los medicamentos que pueden tener uso en esta patología son acetaminofén, antiinflamatorios no esteroides (AINE) selectivos y no selectivos, antidepresivos, anticonvulsivantes, neuromoduladores y opioides.

El acetaminofén y los AINE tradicionales y selectivos son más efectivos que el placebo en relación con el control de dolor oncológico; no hay hasta el momento evidencia que muestre la superioridad de un AINE en particular37; así mismo, hay discrepancia en la literatura disponible respecto a la superioridad de la administración del opioide en combinación con AINE o acetaminofén, dado que no se ha evidenciado disminución en nivel de dolor o consumo total de opioide al usar estas combinaciones ${ }^{38}$; cabe mencionar que algunos estudios favorecen la elección del ketorolaco como terapia aditiva ${ }^{39}$. A pesar de la falta de evidencia, estas combinaciones son una práctica habitual en los servicios de dolor dentro de los esquemas de analgesia multimodal ${ }^{40}$.

Los antidepresivos y anticonvulsivantes son medicamentos comúnmente usados como adyuvantes analgésicos en dolor oncológico; ya hemos visto que el dolor óseo metastásico tiene componente neuropático y además su pobre control favorece fenómenos de sensibilización central. Los antidepresivos y anticonvulsivantes tienen también otros efectos, como ser estabilizadores de ánimo (efecto antidepresivo y ansiolítico) e inductores de sueño, que son benéficos en estos pacientes; cabe resaltar que el efecto de alivio de dolor es independiente del efecto antidepresivo, ya que el efecto analgésico se presenta ante dosis menores y en ausencia de depresión ${ }^{41}$.

De todos los antidepresivos, son los tricíclicos (TCA) los que han mostrado mayor eficacia en control de dolor; en dolor neuropático, la amitriptilina tiene un NNT 1,442; otros grupos de antidepresivos, como los duales selectivos venlafaxina, desvenlafaxina y duloxetina, han mostrado efecto benéfico, por ejemplo, para control de polineuropatía dolorosa, venlafaxina presenta NNT 5,243. Los inhibidores selectivos de recaptación de hidroxitriptamina (5HT), como citalopram, fluoxetina y paroxetina, no han mostrado ser efectivos en comparación con los TCA ${ }^{44}$. Anticonvulsivantes como carbamazepina, oxcarbamazepina, topiramato y lamotrigina han mostrado efectividad analgésica en neuralgia del trigémino, no hay literatura que favorezca su uso en otros tipos de dolor neuropático relacionados con cáncer ${ }^{45,46}$.

Anticonvulsivantes como gabapentino y pregabalina han demostrado ser útiles en el manejo de dolor neuropático relacionado y no relacionado con cáncer ${ }^{47}$; así mismo, la combinación de opioide más antidepresivos y antiepilépticos provee una mejor analgesia que cada uno de ellos por separado ${ }^{48}$. La combinación de pregabalina a dosis bajas de 25 y 50 mg en combinación con antidepresivos (mirtazapina) y opioide (morfina) mostró ser efectiva en el control de dolor y baja incidencia de efectos secundarios en un estudio ${ }^{49}$.

La ketamina es un conocido agente anestésico que en dosis subanestésicas se considera un analgésico adyuvante en el tratamiento de dolor oncológico; sin embargo, no existe evidencia sólida que avale su uso rutinario en dolor óseo metastásico ${ }^{50}$.

Los opioides se consideran medicamentos de elección en pacientes con dolor moderado a severo. Opioides débiles como codeína y tramadol han mostrado ser menos efectivos en el control del síntoma al ser comparados con otros opioides como morfina y fentanilo, y se han asociado a mayor incidencia de 
efectos secundarios gastrointestinales ${ }^{51}$. Existen factores individuales y poblacionales que afectan la sensibilidad a algunos opioides, como morfina, por lo cual es importante tener en cuenta la fármaco-genómica poblacional si esta existiese, así como identificar de forma oportuna fenómenos de tolerancia opioide, de modo que se realice la rotación a otro opioide para lograr el adecuado control del dolor; en este sentido, la morfina y oxicodona se consideran medicamentos equipotentes en control de dolor oncológico ${ }^{52}$.

Nuevos medicamentos opioides, como el tapentadol, han mostrado beneficio en control de dolor oncológico moderado a severo, incluyendo dolor por enfermedad ósea metastásica, con menor incidencia de efectos secundarios gastrointestinales y, por lo tanto, mejor tolerabilidad ${ }^{53}$. Para el manejo del dolor episódico, se prefieren aquellos medicamentos que ofrecen sistemas de liberación rápida que permitan un inicio de acción casi inmediata; dentro de este perfil farmacológico encontramos la morfina de liberación inmediata; sin embargo, los metaanálisis no ofrecen información concluyente que favorezca su uso debido a la pobre calidad de los estudios ${ }^{54}$. Por otro lado, encontramos los sistemas de liberación transmucosa, como el oral y nasal del fentanilo, con mayor efectividad frente a morfina y oxicodona de liberación inmediata ${ }^{55-57}$.

\section{Terapia farmacológica antiosteoclástica Denosumab}

El denosumab es un anticuerpo monoclonal $\lg G 2$ anti-RANKL; reduce la diferenciación, la actividad y la supervivencia de osteoclastos; tiene un amplio rango de acción en entidades patológicas donde se involucran los osteoclastos, incluyendo la osteoporosis, la artritis reumatoidea y el cáncer metastásico. La Sociedad Americana de Oncología Clínica (ASCO) recomienda su uso en pacientes con metástasis ósea con evidencia de destrucción, secundarias a cáncer de mama, y, recientemente, fue aprobado por la FDA para el tratamiento de metástasis óseas por tumores sólidos. La dosis recomendada es de $120 \mathrm{mg}$ por vía subcutánea cada cuatro semanas ${ }^{58}$. Los efectos secundarios más comunes son el dolor lumbar y óseo generalizado, y además es importante considerar el riesgo de osteonecrosis de mandíbula; probablemente sus mayores virtudes son la posibilidad de uso en paciente con falla renal y la facilidad de administración, ya que es subcutánea. Ha demostrado ser no inferior frente a los bifosfonatos en el control de eventos óseos y dolor en carcinomas de mama, próstata y otros tumores ${ }^{59-61}$, aunque no se ha demostrado que mejore la supervivencia global62.

\section{Bifosfonatos}

Los bifosfonatos son medicamentos que inhiben la resorción ósea osteoclástica (Rogers, 1997); se incorporan rápidamente al hueso y se liberan durante la resorción mediada por osteoclastos, deterioran la capacidad de los osteoclastos para adherirse a la superficie ósea, inhibiendo aún más la resorción ósea. Los bisfosfonatos también disminuyen el desarrollo de células precursoras de osteoclastos e inducen la apoptosis de los osteoclastos. Mediante la inhibición de la farnesil difosfato sintasa, una enzima clave en la ruta biosintética de mevalonato. Afectan la resorción ósea a través de la inhibición de la maduración de los precursores de osteoclastos, inhibición de la adhesión de células tumorales al hueso y la inhibición de producción de citoquinas pro inflamatorias. Los bifosfonatos que contienen nitrógeno (N-BPS) tienen la mayor actividad antirresortiva, el ácido zoledrónico es el aminobifosfonato más potente y es el único bisfosfonato intravenoso eficaz en todos los tipos de lesión ósea metastásica ${ }^{58}$.

El pamidronato, zolendronato, ibandronato endovenosos han mostrado que, asociados a quimioterapia u hormonoterapia, son eficaces para reducir el dolor óseo, incidencia y tasa de eventos esqueléticos, y retardar los eventos esqueléticos en mujeres con cáncer de mama avanzado y metástasis óseas clínicamente observables. A su vez, el zolendronato e ibandronato endovenosos han demostrado mejoría en calidad de vida de estas pacientes. El ibandronato oral administrado diariamente, además de la quimioterapia o el tratamiento hormonal, es eficaz en reducción de dolor óseo, tasa de eventos esqueléticos y mejoría de la calidad de vida global63.

El estudio ZICE (estudio de no inferioridad) comparó la administración de ibandronato oral versus zoledronato sin demostrar que el ibandronato fuera no inferior a zoledronato para el manejo de metástasis ósea por cáncer de mama ${ }^{64}$. El estudio ZOOM también aclaró la forma de administrar el zoledronato, definiéndose que, después de nueve meses de tratamiento mensual, es posible continuar con administración de este cada 12 semanas para el tratamiento de cáncer de mama ${ }^{65}$. En el estudio CAGB 90202, se evaluó la utilidad de 
adicionar tempranamente el uso de zoledronato al manejo de cáncer de próstata, junto con terapia de deprivación androgénica (ADT), encontrándose que la adición no disminuyó los eventos esqueléticos, por lo cual la recomendación de guías como NCCN es utilizar bifosfonatos solo después de progresión con ADT ${ }^{66}$.

\section{Terapia con radionucleótidos}

Son útiles para manejo de metástasis ósea poliostótica, especialmente enfermedad con importante actividad osteoblástica; otro posible escenario es como alternativa en aquel paciente en quien la radioterapia está contraindicada, o que tiene mal control del dolor a pesar de haberla recibido. Las contraindicaciones relativas son: enfermedad sistémica no controlada, menos de tres metástasis óseas, metástasis osteolíticas puras, alto riesgo de mielotoxicidad por pobre reserva medular, expectativa de vida menor a 60 días, y las absolutas son: compresión medular, alto riesgo de fractura o fracturas críticas, falla renal, embarazo y lactancia. Las terapias más utilizadas son estroncio-clorhidrato-89 (Sr-89), samario lexidronam-153 (Sm-153), renio-186 (Re-186) y radium223 (Ra-223). El fósforo-32 P ya no es utilizado por alto riesgo de mielotoxicidad 67,68 . En general, el mecanismo de acción está relacionado con daño del DNA de células tumorales y células inflamatorias, con muerte celular subsecuente y disminución de la producción de sustancias alogógenas.

Después de la administración del radiofármaco, la posibilidad de incorporación a la matriz ósea en formación dependerá de la actividad osteoblástica; por esto, es importante definir la extensión y las características de la enfermedad con gammagrafía ósea; la excepción a esta regla es el estroncio y radium-223, ya que tienen propiedades físico-químicas similares al calcio, por lo cual pueden ingresar al hueso directamente. La radiación liberada localmente es de tipo beta, pero el samarium y renio también liberan radiación gamma, lo que permite realizar imágenes, y el radium-223 emite radiación alfa. El Ra-223 retrasa la presentación del primer evento óseo y mejora la supervivencia global en pacientes con cáncer de próstata resistente a la radiación ${ }^{69}$. La eficacia de Ra223 en cáncer de mama metastásico a hueso ha sido demostrada en modelo murino ${ }^{70}$.

La mayoría de los estudios que evalúan la utilidad de radionucleótidos han sido desarrollados en cáncer de próstata ${ }^{71-73}$. Algunos estudios han involucrado pacientes con cáncer de mama ${ }^{74,75}$; en un análisis, el 92\% de los pacientes con cáncer de mama con metástasis ósea y dolor refractario respondieron al manejo con Sr-8976, generalmente el dolor mejora 1-3 semanas, y es importante tener en cuenta que dos días luego de la administración el dolor óseo puede empeorar. El Re-186 tiene menor riesgo de mielosupresión que el Sr-8977. El uso repetido es posible y seguro en pacientes que presentaron mejoría desde el inicio del tratamiento ${ }^{78,79}$. El evento adverso más frecuente es la mielotoxicidad, predomina la trombocitopenia, seguido por anemia y neutropenia. Existen dudas sobre la seguridad del uso de bifosfonatos y radionucleótidos de forma combinada, aunque hay evidencia reciente que favorece su implementación ${ }^{80}$.

\section{Conclusión}

El dolor óseo asociado a enfermedad metastásica tiene una alta incidencia y genera importante morbilidad en los pacientes con cáncer. Los mecanismos de generación del dolor son múltiples y se ha avanzado en el conocimiento de sus aspectos moleculares, sin embargo, la extrapolación de estos a la práctica clínica aún es limitada y las herramientas terapéuticas siguen siendo restringidas. El manejo debe ser individualizado y multidisciplinario, la participación de especialistas en oncología, dolor y cuidados paliativos, radioterapia, rehabilitación oncológica y ortopedia oncológica, entre otros, busca ofrecer el mejor y el más razonable abordaje disponible.

Dentro de las opciones de terapia farmacológica, se encuentran analgésicos comunes, analgésicos opioides, anticonvulsivantes, neuromoduladores y antidepresivos; el uso de bifosfonatos, denosumab, radioterapia, radioablación y radionucleótidos amplía las estrategias aportando un importante beneficio a este grupo de pacientes. Aunque el manejo de esta enfermedad es similar para los diversos tipos de cánceres que generan las metástasis, existen algunas consideraciones particulares, como la falta de utilidad del inicio temprano de bifosfonatos en pacientes con cáncer de próstata sensible a la terapia de deprivación androgénica; la posibilidad de continuar tratamiento cada tres meses con zoledronato luego de nueve meses de tratamiento mensual en pacientes con diagnóstico de cáncer de mama; y la gran utilidad de radium-223 en cáncer de próstata con beneficio positivo en supervivencia global. 


\section{Referencias}

1. Damron T. Oncology and basic science. (s. I.): Lippincott Williams \& Wilkins; 2008

2. Pachman DR, Barton DL, Swetz KM, Loprinzi CL. Troublesome symptoms in cancer survivors: fatigue, insomnia, neuropathy, and pain. J Clin Oncol 2012;30(30):3687-96.

3. Van den Beuken-van Everdingen $\mathrm{MH}$, de Rijke JM, Kessels AG Schouten HC, van Kleef M, Patijn J. Prevalence of pain in patients with cancer: a systematic review of the past 40 years. Ann Oncol 2007;18(9):1437-49.

4. Goudas LC, Bloch R, Gialeli-Goudas M, Lau J, Carr DB. The epidemiology of cancer pain. Cancer Invest 2005;23(2):182-90.

5. Epidemiología del dolor provocado por el cáncer. IASP Año Global del Dolor Producido por el Cáncer 2008-2009. Disponible en: http://www.iasppain.org/files/Content/ContentFolders/GlobalYearAgainstPain2/MusculoskeletalPainFactSheets/Epidemiology_Spanish.pdf

6. Cleeland CS, Gonin R, Hatfield AK, Edmonson JH, Blum RH, Stewart JA, et al. Pain and its treatment in outpatients with metastatic cancer. N Engl J Med 1994;330(9):592-6.

7. Mercadante S. Malignant bone pain: pathophysiology and treatment. Pain 1997;69(1-2):1-18.

8. Gear RW, Miaskowski C, Gordon NC, Paul SM, Heller PH, Levine JD. Kappa-opioids produce significantly greater analgesia in women than in men. Nat Med 1996:2(11):1248-50.

9. World Health Organization. Palliative care: symptom management and end-of-life care: interim guidelines for first-level facility health workers. Geneva; 2004

10. Hoskin P. Opioids in context: relieving the pain of cancer. The role of comprehensive cancer management. Palliat Med 2008;22(4):303-9.

11. Cleeland CS, Janjan NA, Scott CB, Seiferheld WF, Curran WJ. Cancer pain management by radiotherapists: a survey of radiation therapy oncology group physicians. Int J Radiat Oncol Bio Phys 2000;47(1):203-8.

12. Dalal S, Bruera E. Access to opioid analgesics and pain relief for patients with cancer. Nat Rev Clin Oncol 2013;10(2):108-16.

13. Pain and Policy Studies Group. Disponibilidad de analgésicos opioides en las Américas y en el mundo. University of Wisconsin Pain and Policy studies Group/WHO Collaboratin Center for Policy and Communications in Cancer Care, Madison, Wisconsin, EE. UU. Preparado para el I Congreso de la Asociación Latinoamericana de Cuidados Paliativos, VII Curso Latinoamericano de Medicina y Cuidados Paliativos, Guadalajara, México, 20-22 marzo 2002 (Monograph)

14. Harris K, Li K, Flynn C, Chow E. Worst, average or current pain in the Brief Pain Inventory: which should be used to calculate the response to palliative radiotherapy in patients with bone metastases? Clin Oncol (R Coll Radiol) 2007;19(7):523-7.

15. Langford DJ, Tripathy D, Paul SM, West C, Dodd MJ, Schumacher $\mathrm{K}$, et al. Trajectories of pain and analgesics in oncology outpatients with metastatic bone pain. J Pain 2011:12(4):495-507.

16. Cleeland CS. The measurement of pain from metastatic bone disease: capturing the patient's experience. Clin Cancer Res 2006:12:6236s-6242s

17. Puebla F. Tipos de dolor y escala terapéutica de la OMS. Dolor iatrogénico. Oncología 2005:28(3):139-43.
18. Mantyh PW. Substance $P$ and the inflammatory and immune response. Ann N Y Acad Sci 1991;632:263-71.

19. Mantyh PW. Un enfoque mecanístico del dolor oncológico. Rev Soc Esp Dolor 2002;9:481-4

20. Honore P, Rogers SD, Schwei MJ, Salak-Johnson JL, Luger NM, Sabino MC, et al. Murine models of inflammatory, neuropathic and cancer pain each generates a unique set of neurochemica changes in the spinal cord and sensory neurons. Neuroscience 2000;98(3):585-98.

21. Mercadante S, Arcuri E. Breakthrough pain in cancer patients: pathophysiology and treatment. Cancer Treat Rev 1998;24(6):425-32.

22. Nagy I, Rang $H$. Noxious heat activates all capsaicin-sensitive and also a sub-population of capsaicin-insensitive dorsal root ganglion neurons. Neuroscience 1999;88(4):995-7.

23. Basbaum Al. Immediate-early genes and pain: what's all the 'Fos' about? APS J 1994;3:49-52.

24. Armstrong AJ, Creel P, Turnbull J, Moore $C$, Jaffe TA, Haley $S$, et al. A phase I-II study of docetaxel and atrasentan in men with castration-resistant metastatic prostate cancer. Clin Cancer Res 2008;14(19):6270-6.

25. Quinn DI, Tangen CM, Hussain M, Lara PN Jr, Goldkorn A, Moinpour CM, et al. Docetaxel and atrasentan versus docetaxel and placebo for men with advanced castration-resistant prostate cancer (SWOG S0421): a randomised phase 3 trial. Lancet Oncol 2013;14(9):893-900.

26. Muralidharan A, Smith MT. Pathobiology and management of prostate cancer-induced bone pain: recent insights and future treatments. Inflammopharmacol 2013;21(5):339-63.

27. Mach DB, Rogers SD, Sabino MC, Luger NM, Schwei MJ, Pomonis JD, et al. Origins of skeletal pain: sensory and sympathetic innervation of the mouse femur. Neuroscience 2002;113(1):155-66.

28. Mantyh P. Pathophysiology of bone cancer pain. Pain an Update Review 2005

29. Hoskin PJ, Ford HT, Harmer CL. Hemibody irradiation (HBI) for metastatic bone pain in two histological distinct groups of patients. Clin Oncol (R Coll Radiol) 1989;1(2):67-9.

30. Hoskin PJ, Stratford MR, Folkes LK, Regan J, Yarnold JR. Effect of local radiotherapy for bone pain on urinary markers of osteoclast activity. Lancet 2000;355(9213):1428-9.

31. Janjan N, Lutz ST, Bedwinek JM, Hartsell WF, Ng A, Pieters RS Jr, et al. Therapeutic guidelines for the treatment of bone metastasis: a report from the American College of Radiology Appropriateness Criteria Expert Panel on Radiation Oncology. J Palliat Med 2009;12(5):417-26

32. McQuay HJ, Collins S, Carroll D, Moore RA. Radiotherapy for the palliation of painful bone metastases. Cochrane Database Syst Rev 2000;(2):CD001793.

33. Lutz ST, Chow EL, Hartsell WF, Konski AA. A review of hypofractionated palliative radiotherapy. Cancer 2007;109(8):1462-70.

34. Pal S, Dutta S, Adhikary SS, Bhattacharya B, Ghosh B, Patra NB. Hemi body irradiation: an economical way of palliation of pain in bone metastasis in advanced cancer. South Asian J Cancer 2014;3(1):28-32

35. Dupuy DE, Liu D, Hartfeil D, Hanna L, Blume JD, Ahrar K, et al. Percutaneous radiofrequency ablation of painful osseous metastases: a multi-center American College of Radiology Imaging Network trial. Cancer 2010;116(4):989-97. 
36. Goetz MP, Callstrom MR, Charboneau JW, Farrell MA, Maus TP, Welch TJ, et al. Percutaneous image-guided radiofrequency ablation of painful metastases involving bone: a multicenter study. J Clin Oncol 2004;22(2):300-6.

37. Nabal M, Librada S, Redondo MJ, Pigni A, Brunelli C, Caraceni A. The role of paracetamol and nonsteroidal anti-inflammatory drugs in addition to WHO Step III opioids in the control of pain in advanced cancer. A systematic review of the literature. Palliat Med 2012;26(4):305-12.

38. Mercadante $S$, Giarratano A. The long and winding road of non steroidal antinflammatory drugs and paracetamol in cancer pain management: a critical review. Crit Rev Oncol Hematol 2013;87(2):140-5.

39. Moselli NM, Cruto M, Massucco P, Savojardo M, Debernardi F. Long-term continuous subcutaneous infusion of ketoprofen combined with morphine: a safe and effective approach to cancer pain. Clin J Pain 2010;26(4):267-74.

40. McNicol E, Strassels SA, Goudas L, Lau J, Carr DB. NSAIDS or paracetamol, alone or combined with opioids, for cancer pain. Cochrane Database Syst Rev 2005;(1):CD005180

41. McQuay HJ, Carroll D, Glynn CJ. Low dose amitriptyline in the treatment of chronic pain. Anaesthesia 1992;47(8):646-52.

42. Sindrup SH, Jensen TS. Efficacy of pharmacological treatments of neurophatic pain: an update and effect related to mechanism of drug action. Pain 1999;83(3):389-400.

43. Sindrup SH, Bach FW, Madsen C, Gram LF, Jensen TS. Venlafaxine versus imipramine in painful polyneuropathy: a randomized, controlled trial. Neurology 2003;60(8):1284-9.

44. McQuay HJ, Tramer M, Nye BA, Carroll D, Wiffen PJ, Moore RA. A systematic review of antidepressants in neuropathic pain. Pain 1996;68(2-3):217-27.

45. Krafft RM. Trigeminal neuralgia. Am Fam Physician 2008;77(9):1291-6.

46. Buescher JJ. Carbamazepine for acute and chronic pain. Am Fam Physician 2006;73(9):1549-50.

47. Finnerup NB, Sindrup SH, Jensen TS. The evidence for pharmacological treatment of neuropathic pain. Pain 2010;150(3):573-81.

48. Arai YC, Matsubara T, Shimo K, Suetomi K, Nishihara M, Ushida T, et al. Low-dose gabapentin as useful adjuvant to opioids for neuropathic cancer pain when combined with low-dose imipramine. J Anesth 2010;24(3):407-10.

49. Nishihara $M$, Arai $Y C$, Yamamoto $Y$, Nishida K, Arakawa M, Ushida T, et al. Combinations of low-dose antidepressants and lowdose pregabalin as useful adjuvants to opioids for intractable, painful bone metastases. Pain Physician 2013;16(5):E547-52.

50. Bell RF, Eccleston C, Kalso EA. Ketamine as an adjuvant to opioids for cancer pain. Cochrane Database Syst Rev 2012;11:CD003351.

51. Koyyalagunta $D$, Bruera E, Solanki DR, Nouri $K H$, Burton AW, Toro $M P$, et al. A systematic review of randomized trials on the effectiveness of opioids for cancer pain. Pain Physician 2012;15:ES39-58.

52. Riley J, Branford R, Droney J, Gretton S, Sato H, Kennett A, et al. Morphine or oxycodone for cancer-related pain? A randomized, open-label, controlled trial. J Pain Symptom Manage 2015;49(2):161-72

53. Kress HG, Koch ED, Kosturski H, Steup A, Karcher K, Lange $B$, et al. Tapentadol prolonged release for managing moderate to severe, chronic malignant tumor-related pain. Pain Physician 2014;17(4):329-43
54. Wiffen PJ, Wee B, Moore RA. Oral morphine for cancer pain. Cochrane Database Syst Rev 2013;7:CD003868.

55. Coluzzi PH, Schwartzberg L, Conroy JD, Charapata S, Gay M, Busch MA, et al. Breakthrough cancer pain: a randomized trial comparing oral transmucosal fentanyl citrate (OTFC) and morphine sulfate immediate release (MSIR). Pain 2001;91(1-2):123-30.

56. Zeppetella G, Davies AN. Opioids for the management of breakthrough pain in cancer patients. Cochrane Database Syst Rev 2013;10:CD004311.

57. Webster LR, Slevin KA, Narayana A, Earl CQ, Yang R. Fentanyl buccal tablet compared with immediate-release oxycodone for the management of breakthrough pain in opioid-tolerant patients with chronic cancer and noncancer pain: a randomized, double-blind, crossover study followed by a 12-week open-label phase to evaluate patient outcomes. Pain Med 2013;14(9):1332-45.

58. Loftus LS, Edwards-Bennett S, Sokol GH. Systemic therapy for bone metastases. Cancer Control 2012;19(2):145-53.

59. Iranikhah $M$, Stricker $S$, Freeman MK. Future of bisphosphonates and denosumab for men with advanced prostate cancer. Cancer Manag Res 2014;6: 217-24.

60. Fizazi $K$, Carducci $M$, Smith $M$, Damião R, Brown J, Karsh $L$, et al. Denosumab versus zoledronic acid for treatment of bone metastases in men with castration-resistant prostate cancer: a randomised, double-blind study. Lancet 2011:377(9768):813-22.

61. Stopeck AT, Lipton A, Body JJ, Steger GG, Tonkin K, de Boer $\mathrm{RH}$, et al. Denosumab compared with zoledronic acid for the treatment of bone metastases in patients with advanced breast cancer: a randomized, double-blind study. J Clin Oncol 2010;28(35):5132-9.

62. Henry DH, Costa L, Goldwasser F, Hirsh V, Hungria V, Prausova $\mathrm{J}$, et al. Randomized, double-blind study of denosumab versus zoledronic acid in the treatment of bone metastases in patients with advanced cancer (excluding breast and prostate cancer) or multiple myeloma. J Clin Oncol 2011;29(9):1125-32.

63. Pavlakis N, Schmidt RL, Stockler M. Bifosfonatos para el cáncer de mama (revisión Cochrane traducida). Biblioteca Cochrane Plus 2006;(2). Oxford: Update Software Ltd. Disponible en: http://www.update-software.com (Traducida de The Cochrane Library 2006;(2). Chichester, UK: John Wiley \& Sons, Ltd.).

64. Barrett-Lee P, Casbard A, Abraham J, Hood K, Coleman R, Simmonds $\mathrm{P}$, et al. Oral ibandronic acid versus intravenous zoledronic acid in treatment of bone metastases from breast cancer: a randomised, open label, non-inferiority phase 3 trial. Lancet Oncol 2014;15(1):114-22.

65. Amadori D, Aglietta M, Alessi B, Gianni L, Ibrahim T, Farina G, et al. Efficacy and safety of 12-weekly versus 4-weekly zoledronic acid for prolonged treatment of patients with bone metastases from breast cancer (ZOOM): a phase 3, open-label, randomised, non-inferiority trial. Lancet Oncol 2013;14(7):663-70.

66. Smith MR, Halabi S, Ryan CJ, Hussain A, Vogelzang N, Stadler $W$, et al. Randomized controlled trial of early zoledronic acid in men with castration-sensitive prostate cancer and bone metastases: results of CALGB 90202 (alliance). J Clin Oncol 2014;32(11):1143-50.

67. Hillegonds DJ, Franklin S, Shelton DK, Vijayakumar S, Vijayakumar $V$. The management of painful bone metastases with an emphasis on radionuclide therapy. J Natl Med Assoc 2007:99(7):785-94 
68. Pandit-Taskar N, Batraki M, Divgi CR. Radiopharmaceutical therapy for palliation of bone pain from osseous metastases. J Nuc Med 2004;45(8):1358-65.

69. Parker C, Nilsson S, Heinrich D, Helle SI, O'Sullivan JM, Fosså $\mathrm{SD}$, et al. Alpha emitter radium-223 and survival in metastatic prostate cancer. N Engl J Med 2013;369(3):213-23.

70. Suominen MI, Rissanen JP, Kakonen R, Fagerlund KM, Alhoniemi $E$, Mumberg $D$, et al. Survival benefit with radium-223 dichloride in a mouse model of breast cancer bone metastasis. J Natl Cancer Inst 2013;105(12):908-16.

71. Oosterhof GO, Roberts JT, de Reijke TM, Engelholm SA, Horenblas S, von der Maase $\mathrm{H}$, et al. Strontium(89) chloride versus palliative local field radiotherapy in patients with hormonal escaped prostate cancer: a phase III study of the European Organisation for Research and Treatment of Cancer, Genitourinary Group. Eur Urol 2003;44(5):519-26.

72. Tu SM, Millikan RE, Mengistu B, Delpassand ES, Amato RJ, Pagliaro LC, et al. Bone-targeted therapy for advanced androgenindependent carcinoma of the prostate: a randomised phase II trial. Lancet 2001;357(9253):336-41.

73. Sartor O, Reid RH, Hoskin PJ, Quick DP, Ell PJ, Coleman RE, et al. Samarium-153-Lexidronam complex for treatment of painful bone metastases in hormone-refractory prostate cancer. Urology $2004 ; 63(5): 940-5$.

74. Fuster D, Herranz D, Vidal-Sicart S, Muñoz M, Conill C, Mateos JJ, et al. Usefulness of strontium-89 for bone pain pallia- tion in metastatic breast cancer patients. Nucl Med Commun 2000;21(7):623-6.

75. Baczyk M, Czepczynski R, Milecki P, Pisarek M, Oleksa R, Sowinsk J. 89Sr versus 153Sm-EDTMP: comparison of treatment efficacy of painful bone metastases in prostate and breast carcinoma. Nucl Med Commun 2007:28(4):245-50.

76. Fuster D, Herranz D, Vidal-Sicart S, Muñoz M, Conill C, Mateos JJ, et al. Usefulness of strontium-89 for bone pain palliation in metastatic breast cancer patients. Nucl Med Commun 2000;21(7):623-6.

77. Sciuto R, Festa A, Pasqualoni R, Semprebene A, Rea S, Bergomi $S$, et al. Metastatic bone pain palliation with 89-Sr and 186-Re-HEDP in breast cancer patients. Breast Cancer Res Treat 2001;66(2):101-9.

78. Englaro EE, Schroder LE, Thomas SR, Williams CC, Maxon HR 3rd. Safety and efficacy of repeated sequential administrations of Re-186(Sn)HEDP as palliative therapy for painful skeletal metastases. Initial case reports of two patients. Clin Nucl Med 1992;17(1):41-4

79. Sartor O, Reid RH, Hoskin PJ, Quick DP, Ell PJ, Coleman RE, et al. Samarium-153-Lexidronam complex for treatment of painful bone metastases in hormone-refractory prostate cancer. Urology 2004;63(5):940-5.

80. Rubini G, Nicoletti A, Rubini D, Asabella AN. Radiometabolic treatment of bone-metastasizing cancer: from 186rhenium to 223radium. Cancer Biother Radiopharm 2014;29(1):1-11. 